\title{
Condición fisiológica de una población de Arca zebra (Bivalvia: Arcidae) del banco de Chacopata, Sucre, Venezuela
}

\author{
José Villarroel ${ }^{1}$, Vanessa Acosta $^{* 1}$ \& Dwight Arrieche ${ }^{2}$ \\ 1. Departamento de Biología, Núcleo de Sucre, Universidad de Oriente, Cumaná, Estado Sucre. 6101, Venezuela; \\ javm.21@gmail.com \\ 2. Instituto de Investigaciones en Biomedicina y Ciencias Aplicadas Dra. SusanTai, Universidad de Oriente, Cumaná, \\ Estado Sucre, 6101, Venezuela; darrieche@udo.edu.ve, darriech@yahoo.com \\ *Correspondencia: vanessaacosta@yahoo.com
}

Recibido 11-III-2015. C Corregido 08-II-2016. Aceptado 01-III-2016.

\begin{abstract}
Physiological condition of an Arca zebra (Bivalvia: Arcidae) population from Chacopata, Sucre, Venezuela. Condition indices reflect the performance of an organism in their environment, as well as their somatic condition, nutritional value, commercial importance, the ecological role and fattening periods. In this study, different physiological indices were evaluated in order to know the Arca zebra population status, and to suggest operation and sustainability strategies for this resource use and consumption. A total of 240 samples ( 82 males and 158 females) were collected between July 2010 and July 2011, from the Chacopata bank site. Bivalve biometric measurements were made monthly and considered: total shell length, height and width; besides, soft tissues were dehydrated to obtain total dry mass. Different condition indices were analyzed based on the body mass, or on the combination of mass and shell length: gonadal index, muscle index, meat yield, the fresh mass of soft tissue in relation to the total length, and dry mass of soft tissue relative to the total length. We found the model with positive allometric growth between the length-total dry mass. The gonadal weight exerted a marked influence on the condition index, gonadosomatic index and meat yield. Increases in soft tissue mass were proportional to the dimensions of the shell. The index contrasts fresh mass of soft tissue in relation to the total length, as a tool that would allow fishers to estimate the population dynamics of $A$. zebra, and to practice a sustainable fishery over time, while keeping the extraction canons. Rev. Biol. Trop. 64 (3): 1345-1352. Epub 2016 September 01.
\end{abstract}

Key words: condition index, population dynamics, spawning, energy storage.

Actualmente, en los bivalvos de importancia comercial, es común el uso de índices fisiológicos, que ayudan a estimar el estado de bienestar y desarrollo de los individuos, el valor económico para su explotación y consumo, y además, permiten conocer la respuesta del organismo ante variaciones ambientales (Acosta, Prieto, \& Lodeiros, 2006). La literatura reporta diferentes métodos para estimar la condición fisiológica, desde el uso de los datos biométricos, hasta procedimientos bioquímicos que son más costosos, que requieren más tiempo y tienen la ventaja de dar información precisa de porciones del organismo y órganos. Sin embargo, el estado fisiológico de una población, refleja la tendencia promedio de los individuos de la proporción de tejidos en relación al tamaño de la concha, masa o gónadas. En este sentido, estos indicadores ayudan a predecir los cambios que ocurren en el desempeño de la condición somática, valor nutritivo, períodos de engorde (Cruz, 1982; Prieto, Ramos, Arrieche, Villalba, \& Lodeiros, 2001; Aponte, Prieto, \& Lemus, 2008), estado reproductivo, transferencia de energía (Lucas \& Beninger 1985; Acosta et al., 2006; Acosta, Prieto, Licett, Longart, \& Montes, 2011) y el efecto ambiental sobre el organismo en un momento dado (Sarkis, 1992; 1993).

Arca zebra, es un bivalvo perteneciente a la familia Arcidae, habita fondos arenosos de grava, formando grupos adheridos por el biso. 
En Venezuela, los principales bancos naturales y explotados comercialmente se localizan en la zona nororiental, al este de la Isla de Margarita y el eje Coche-Chacopata, de donde se extrae el $95 \%$ de la producción de moluscos de Venezuela (Salaya, 1999). Es el recurso más importante entre los moluscos del Nororiente de Venezuela con los mayores volúmenes de pesca. Sin embargo, en el último decenio, se ha reportado una disminución significativa de la extracción, lo cual puede representar, a mediano plazo, un problema para la economía regional (Jiménez, 1999; Salaya, 1999; Mendoza, 2008); y ya actualmente, afecta a las empresas enlatadoras que difícilmente reciben la carga mínima para su funcionamiento (Comunic. Pers, César Cabrera草).

La extracción de estos moluscos, se hace según la regulación del Ministerio de Agricultura y Cría (MAC-RNR-266 de fecha 02/09/1960), que establece una talla mínima de $30 \mathrm{~mm}$ de grosor (Gr) de la concha, que equivale a $60 \mathrm{~mm}$ de longitud total $(\mathrm{Lt})$, tamaño con el cual ha alcanzado la madurez sexual. Hasta ahora, a pesar de conocerse su ciclo reproductivo (Lista et al., 2011), la calidad y cantidad de carne de A. zebra oscila estacionalmente, $\mathrm{y}$ al no tener dimorfismo sexual, sus gónadas son difíciles de apreciar externamente. En este trabajo, se evaluaron algunos índices de condición, con el propósito de explicar la dinámica poblacional mensual y entre tallas $(\mathrm{Lt}) \mathrm{del}$ banco de Chacopata, con organismos que cumplen el criterio de extracción para el consumo y comercialización, con el propósito de contribuir al conocimiento de la dinámica poblacional, su relación con los períodos de engorde, y variables ambientales que caracterizan a la región por su alta productividad primaria.

\section{MATERIALES Y MÉTODOS}

Obtención de los organismos: Se recolectaron 240 ejemplares entre julio 2010 y julio 2011, en el banco de Chacopata (10 $42^{\circ}-10^{\circ} 46^{\prime}$ N y $\left.63^{\circ} 46^{\prime}-63^{\circ} 54^{\prime} \mathrm{W}\right)$, estado Sucre, procedentes de la pesquería comercial, que se realiza con embarcaciones equipadas con rastra (120 x $86 \mathrm{~cm}$ ) y que trabajan entre $8-12 \mathrm{~m}$ de profundidad. La selección de los ejemplares siguió la regulación MAC-RNR-266, que permite la extracción de ejemplares con grosor $(\mathrm{Gr})$ de la concha superior a $30 \mathrm{~mm}$. Los organismos fueron trasladados en contenedores isotérmicos y se congelaron.

Variables biométricas: En el laboratorio, se removieron los epibiontes antes de realizar las mediciones biométricas. Se midió en la concha la distancia antero-posterior (Longitud total, Lt), la mayor distancia perpendicular a la longitud (Altura, $\mathrm{Al}$ ) y la mayor distancia transversal a Lt (Grosor, Gr) (vernier \pm 0.01 $\mathrm{mm}$ ). Se determinó el sexo por la coloración de las gónadas, y se identificó a las hembras de color marrón-naranja $(\mathrm{n}=82)$ y blanquecino a los machos ( $\mathrm{n}=158)$ (Lista et al., 2006; 2011). Se obtuvo la masa total (Mt) libre de epibiontes por la suma de la masa de la concha (Mc), la masa de los tejidos blandos frescos escurridos $(\mathrm{Mh}=\mathrm{Mhm}+\mathrm{Mhg}+\mathrm{Mhr})$ que se disectaron en músculo $(\mathrm{Mhm})$, gónada (Mhg) y resto de tejidos (Mhr) $( \pm 0.001 \mathrm{~g})$; estos fueron deshidratados hasta masa seca constante $\left(60^{\circ} \mathrm{C} / 48 \mathrm{~h}\right)$ y se obtuvo la masa seca total $(\mathrm{Ms}=\mathrm{Msm}+\mathrm{Msg}$ + Msr) por la suma de la masa seca del músculo (Msm), gónada (Msg) y resto de tejidos (Msr).

Estructura de la población: Se determinaron las cohortes por el método probabilístico de Harding (1949), se graficó Lt con la frecuencia porcentual acumulativa de Lt (\%) en papel probabilístico, y se ubicaron los puntos de inflexión que separan las cohortes.

Índices de condición fisiológica: Se obtuvieron diferentes modelos de condición fisiológica a partir de las variables biométricas; masa total (Mt), masa fresca de los tejidos blandos (Mh), masa de la concha (Mc), masa seca; de los tejidos blandos (Ms), gónada (Msg), músculo (Msm) y Lt: $\mathrm{IC}_{1}=100 \mathrm{x}(\mathrm{Mh} /$ Mc) (Nascimento \& Pereira, 1980), $\mathrm{IC}_{2}=100$ $\mathrm{x}(\mathrm{Ms} / \mathrm{Mc})$ (Davenport \& Chen, 1987); índice gonadal (IG) $\mathrm{IG}=100 \times(\mathrm{Msg} / \mathrm{Ms})($ Hickman \& Illingworth, 1980); índice muscular (IM): 
$\mathrm{IM}=100 \times(\mathrm{Msm} / \mathrm{Ms})$ (Barber \& Blake, 1991); rendimiento $(\mathrm{R}) \mathrm{R}=100 \times(\mathrm{Mh} / \mathrm{Mt})$, relación entre la masa húmeda con $\mathrm{Lt}\left(\mathrm{IC}_{3}=100 \mathrm{x}(\mathrm{Mh} /\right.$ Lt) (Kagley, Snider, Krishnakumar, \& Casillas, 2003) y la relación entre la masa seca con la longitud de la concha $\left(\mathrm{IC}_{4}=100 \times(\mathrm{Ms} / \mathrm{Lt})\right.$ (Martin, Ichikawa, Goetzl, Reyes, \& Stephenson, 1984). Se aplicó el modelo de crecimiento alométrico con transformación logarítmica de la longitud total (Lt) y la masa seca de los tejidos blandos $\left(\mathrm{Ms}=\mathrm{aLt}^{\mathrm{b}}\right)$.

Variables ambientales: Quincenalmente in situ se midió la temperatura $\left({ }^{\circ} \mathrm{C}\right)$, se recolectó agua de mar en botellas Niskin (2L) entre 8-12 m de profundidad. Se midió la salinidad ( \pm 1 UPS), la clorofila $a(\mu \mathrm{g} / / \mathrm{L})$ y el seston total (mg/L) (Strickland \& Parsons, 1972).

Se comprobó la homogeneidad de las varianzas de las variables ambientales, variables biométricas e índices de condición. Las variables biométricas de la concha se examinaron con un modelo de regresión lineal, para determinar la variable con la correlación más significativa. Los datos se examinaron entre meses e intervalos de longitud total de la concha $( \pm 10 \mathrm{~mm})$. Entre sexos no se detectaron diferencias significativas. Los índices de condición se evaluaron por medio de un análisis de varianza (ANOVA) sencilla entre meses, las diferencias significativas $(\mathrm{P} \leq 0.05)$ se ajustaron por una prueba a posteriori de Scheffe (Sokal \& Rohlf, 1969). Los promedios con menos de 30 datos se analizaron con una prueba no paramétrica de Kolmogorov-Smirnov (KS) (P $\leq 0.05)$. Los índices de condición, variables ambientales y los períodos de surgencia y estratificación anual se analizaron por un análisis de Análisis de Componentes Principales (ACP).

\section{RESULTADOS}

Estructura poblacional y variables biométricas: La muestra examinada estuvo integrada por una cohorte principal del intervalo 60-79 mm Lt con el $98 \%$ de los organismos. La Lt promedio anual fue de $70.3 \pm 9.6 \mathrm{~mm}$ y de grosor de $32.6 \pm 4.3 \mathrm{~mm}$. Las variables biométricas mensuales presentaron diferencias significativas entre los meses con incrementos periódicos mensuales (Cuadro 1). La Lt se correlacionó positiva y significativamente con la altura (Al) y el grosor de la concha (Gr), ajustado por los modelos lineales de regresión: $\mathrm{Lt}=0.36 \mathrm{Al}+7.13\left(\mathrm{r}^{2}=0.66\right) \mathrm{y} \mathrm{Lt}=0.48 \mathrm{Gr}+$ $2.93\left(r^{2}=0.63\right)$.

Índice de condición fisiológica: Los índices de condición $\left(\mathrm{IC}_{1}, \mathrm{IC}_{3}, \mathrm{IC}_{4}, \mathrm{IG}\right.$ y $\mathrm{R}$ )

CUADRO 1

Promedios mensuales de las variables biométricas de Arca zebra del banco de Chacopata, Venezuela

TABLE 1

Average monthly values of the biometric variables from Arca zebra at Chacopata, Venezuela

\begin{tabular}{cccccccccc} 
Mes & Lt & Alt & Gr & Mt & Mh & Mc & Ms & Msm & Msg \\
J/2010 & $59,9 \pm 2,1^{\text {a }}$ & $29,7 \pm 1,^{\text {a }}$ & $25,6 \pm 0,7^{\mathrm{a}}$ & $24,0 \pm 2,1$ & $7,58 \pm 0,7$ & $16,4 \pm 1,4$ & $1,6 \pm 0$, & $0,2 \pm 0,02$ & $0,4 \pm 0,06$ \\
A & $75,6 \pm 1,8^{\mathrm{b}}$ & $39,5 \pm 0,8^{\mathrm{b}}$ & $36,2 \pm 0,7^{\mathrm{b}}$ & $44,2 \pm 2,3$ & $14,3 \pm 0,8$ & $29,9 \pm 1,6$ & $3,2 \pm 0,2$ & $0,5 \pm 0,03$ & $0,9 \pm 0,07$ \\
$\mathrm{~S}$ & $79,8 \pm 1,0^{\mathrm{b}}$ & $42,37 \pm 0,54^{\mathrm{b}}$ & $37,5 \pm 0,5^{\mathrm{b}}$ & $47,3 \pm 1,3$ & $13,9 \pm 0,5$ & $33,4 \pm 0,9$ & $3,3 \pm 0,2$ & $0,5 \pm 0,03$ & $0,8 \pm 0,1$ \\
O & $60,9 \pm 1,4^{\mathrm{a}}$ & $33,9 \pm 0,5^{\mathrm{a}}$ & $29,6 \pm 0,4^{\mathrm{c}}$ & $30,9 \pm 1,3$ & $9,3 \pm 0,6$ & $21,6 \pm 1,1$ & $1,9 \pm 0,1$ & $0,3 \pm 0,01$ & $0,6 \pm 0,1$ \\
N & $70,1 \pm 1,9^{\mathrm{a}}$ & $34,7 \pm 0,8^{\mathrm{a}}$ & $32,5 \pm 0,6$ & $31,4 \pm 1,9$ & $7,10 \pm 0,5$ & $24,25 \pm 1,6$ & $1,57 \pm 0,12$ & $0,23 \pm 0,02$ & $0,3 \pm 0,06$ \\
D & $73,9 \pm 1,5^{\mathrm{b}}$ & $42,4 \pm 1,2^{\mathrm{b}}$ & $35,0 \pm 0,4^{\mathrm{b}}$ & $45,1 \pm 2,5$ & $10,2 \pm 0,5$ & $34,9 \pm 2,2$ & $2,5 \pm 0,1$ & $0,39 \pm 0,02$ & $0,6 \pm 0,07$ \\
$\mathrm{E} / 2011$ & $71,2 \pm 1,5^{\mathrm{b}}$ & $36,9 \pm 0,9^{\mathrm{b}}$ & $33,7 \pm 0,7$ & $38,8 \pm 1,9$ & $8,8 \pm 0,5$ & $30,0 \pm 1,5$ & $1,8 \pm 0,1$ & $0,3 \pm 0,03$ & $0,3 \pm 0,05$ \\
F & $74,9 \pm 1,9^{\mathrm{b}}$ & $39,7 \pm 1,2^{\mathrm{b}}$ & $33,3 \pm 0,7$ & $39,1 \pm 2,6$ & $7,8 \pm 0,1$ & $31,2 \pm 2,2$ & $2,1 \pm 0,1$ & $04 \pm 0,03$ & $0,1 \pm 0,02$ \\
M & $60 \pm 1,8^{\mathrm{a}}$ & $30,6 \pm 1,2^{\mathrm{a}}$ & $27,8 \pm 0,8^{\mathrm{a}, \mathrm{b}}$ & $22,2 \pm 1,7$ & $6,9 \pm 0,51$ & $15,3 \pm 1,2$ & $1,3 \pm 0,1$ & $0,2 \pm 0,02$ & $0,2 \pm 0,0$ \\
M & $73,9 \pm 1,9^{\mathrm{b}}$ & $38,1 \pm 1,0^{\mathrm{b}}$ & $32,9 \pm 0,6$ & $38,7 \pm 2,5$ & $11,7 \pm 0,8$ & $27,0 \pm 1,8$ & $2,56 \pm 0,18$ & $0,39 \pm 0,04$ & $0,5 \pm 0,06$ \\
J & $70,8 \pm 1,2^{\mathrm{b}}$ & $36,8 \pm 1,0^{\mathrm{b}}$ & $33,2 \pm 0,5$ & $36,3 \pm 1,6$ & $7,1 \pm 0,2$ & $29,1 \pm 1,4$ & $1,62 \pm 0,10$ & $0,3 \pm 0,02$ & $0,24 \pm 0,03$ \\
J & $71,2 \pm 1,5^{\mathrm{b}}$ & $36,9 \pm 0,1^{* \mathrm{~b}}$ & $33,7 \pm 0,7$ & $38,8 \pm 1,9$ & $8,8 \pm 0,51$ & $30,0 \pm 1,5$ & $1,8 \pm 0,1$ & $0,3 \pm 0,03$ & $0,3 \pm 0,05$ \\
\hline
\end{tabular}


presentaron oscilaciones mensuales sincronizadas con diferencias significativas mensuales ( $\mathrm{P} \leq 0.05$; Cuadro 2). El índice muscular no presentó diferencias entre los meses, con tendencia a oscilar en relación inversa al IG. Los índices de condición entre los intervalos de longitud presentaron diferentes tendencias, lo que permite agruparlos de acuerdo a los valores máximos y mínimos de intervalos de tallas y sexos. El $\mathrm{IC}_{1}$ e IG no presentaron diferencias significativas entre las tallas ( $\mathrm{P} \geq 0.05)$, mientras que los $\mathrm{IC}_{1}, \mathrm{IM}, \mathrm{IC}_{3} \mathrm{y} \mathrm{IC}_{4}$ incrementaron con la talla; sin embargo, los tres últimos, presentaron diferencias significativas $(\mathrm{P} \geq 0.05)$ entre los intervalos 50-69 con 70-79, tendencia que se observó en machos y hembras. En otro grupo, se aprecian comportamientos similares del $\mathrm{IC}_{2}, \mathrm{R}$ e IG, que tienen los valores promedio más altos en el intervalo 40-59 mm Lt, seguido de una disminución desde el intervalo 60-69 mm a 90-99 mm (IG), mientras que el $\mathrm{IC}_{2}$ y $\mathrm{R}$ tienden a ser altos en los organismos más grandes (90-99 mm) (Cuadro 2 y Cuadro 3).
Análisis de componentes principales (ACP): El ACP entre los factores ambientales con el rendimiento $(\mathrm{R}), \mathrm{IG}$ e $\mathrm{IC}_{1}$, se relacionó positivamente con la temperatura explicando el $70 \%$ de la varianza acumulada, la clorofila $a$ y el seston total. La clorofila $a$, seston total y la salinidad, no mostraron una asociación positiva con los índices fisiológicos; sin embargo, en marzo, mayo y julio 2011, se asociaron con la productividad primaria procedente de los periodos de surgencia. Se obtuvo una relación negativa entre el IM con el rendimiento (R), índice gonadal (IG) e índice de condición (Fig. 1).

\section{DISCUSIÓN}

Los estudios poblacionales por lo general abarcan los cambios de una comunidad integrada por diferentes grupos etarios, en los cuales la condición fisiológica está sesgada por el sexo, edad, maduración gonadal y condiciones particulares del medio ambiente. Arca zebra es un recurso sujeto a explotación continua,

CUADRO 2

Promedios mensuales de los índices de condición de Arca zebra en el banco Chacopata, Venezuela

TABLE 2

Arca zebra average month condition indices from Chacopata, Venezuela

\begin{tabular}{|c|c|c|c|c|c|c|c|c|c|c|}
\hline \multirow{2}{*}{ Mes } & \multicolumn{3}{|c|}{ Relación alométrica } & \multirow{2}{*}{$\mathrm{IC}_{1}$} & \multirow{2}{*}{$\mathrm{IC}_{2}$} & \multirow{2}{*}{$\mathrm{R}$} & \multirow{2}{*}{ IG } & \multirow{2}{*}{ IM } & \multirow{2}{*}{$\mathrm{IC}_{3}$} & \multirow{2}{*}{$\mathrm{IC}_{4}$} \\
\hline & $\mathrm{a}$ & B & $\mathrm{r}$ & & & & & & & \\
\hline $\mathrm{J} / 2010$ & -1.72 & 1.07 & 0.39 & $20.9 \pm 2.6^{b}$ & $9.8 \pm 2.3^{\mathrm{a}}$ & $31.6 \pm 3.8^{a}$ & $26.1 \pm 9.5^{\mathrm{a}}$ & $12.9 \pm 4.0$ & $12.5 \pm 4.2^{\mathrm{a}}$ & $2.6 \pm 1.0^{\mathrm{a}}$ \\
\hline A & -3.56 & 2.16 & $0.79 *$ & $22.5 \pm 1.9^{\mathrm{a}}$ & $10.9 \pm 2.2^{\mathrm{a}}$ & $32.3 \pm 3.3^{\mathrm{a}}$ & $27.4 \pm 5.0^{\mathrm{a}}$ & $14.0 \pm 2.1$ & $18.7 \pm 3.2^{b}$ & $4.2 \pm 0.9^{b}$ \\
\hline S & -4.38 & 2.57 & $0.53 *$ & $23.8 \pm 3.7^{\mathrm{a}}$ & $10.0 \pm 2.5^{\mathrm{a}}$ & $29.3 \pm 3.4^{\mathrm{a}}$ & $22.3 \pm 8.7^{\mathrm{a}}$ & $15.6 \pm 2.9$ & $17.4 \pm 2.5^{b}$ & $4.2 \pm 1.0^{b}$ \\
\hline $\mathrm{O}$ & -0.39 & 0.37 & $0.16^{*}$ & $20.7 \pm 4.4^{\mathrm{a}, \mathrm{b}}$ & $8.9 \pm 2.4^{\mathrm{a}, \mathrm{b}}$ & $30.2 \pm 6.6^{\mathrm{a}}$ & $28.1 \pm 15.3^{\mathrm{a}}$ & $14.7 \pm 3.8$ & $15.4 \pm 4.7^{b}$ & $3.1 \pm 0.7^{\mathrm{a}}$ \\
\hline $\mathrm{N}$ & 0.20 & 0.28 & 0.53 & $22.7 \pm 6.6^{\mathrm{a}, \mathrm{b}}$ & $6.6 \pm 1.7^{b}$ & $22.7 \pm 3.6^{b}$ & $17.9 \pm 11.4^{b}$ & $15.0 \pm 4.0$ & $10.0 \pm 2.3^{\mathrm{a}}$ & $2.2 \pm 0.6^{\mathrm{a}}$ \\
\hline $\mathrm{D}$ & -2.98 & 1.71 & 0.51 & $24.6 \pm 3.8^{a, b}$ & $7.5 \pm 2.1 \mathrm{a}, \mathrm{b}$ & $23.0 \pm 3.6^{b}$ & $21.1 \pm 10.6^{\mathrm{a}, \mathrm{b}}$ & $15.9 \pm 3.2$ & $13.7 \pm 2.2^{\mathrm{a}}$ & $3.4 \pm 0.7^{\mathrm{a}, \mathrm{b}}$ \\
\hline $\mathrm{E} / 2011$ & -4.14 & 2.36 & $0.67 *$ & $20.1 \pm 2.0^{b}$ & $5.9 \pm 1.0^{\mathrm{b}}$ & $22.6 \pm 2.0^{b}$ & $18.0 \pm 7.8^{b}$ & $17.9 \pm 4.0$ & $12.2 \pm 2.4^{\mathrm{a}}$ & $2.5 \pm 0.7^{\mathrm{a}}$ \\
\hline $\mathrm{F}$ & -3.69 & 2.13 & $0.84 *$ & $28.0 \pm 6.5^{\mathrm{a}}$ & $7.0 \pm 1.6^{\mathrm{a}, \mathrm{b}}$ & $20.3 \pm 4.7^{b}$ & $4.8 \pm 4.5^{b}$ & $18.6 \pm 4.4$ & $10.3 \pm 2.8^{\mathrm{a}}$ & $2.8 \pm 0.5^{\mathrm{a}}$ \\
\hline M & -4.11 & 2.36 & $0.87 *$ & $19.2 \pm 2.6^{\mathrm{b}}$ & $8.8 \pm 1.9^{\mathrm{a}, \mathrm{b}}$ & $31.3 \pm 3.4^{\mathrm{a}}$ & $11.4 \pm 7.0^{b}$ & $15.0 \pm 4.1$ & $11.0 \pm 2.7^{\mathrm{a}}$ & $2.1 \pm 0.5^{a}$ \\
\hline M & -2.73 & 1.67 & $0.62 *$ & $21.9 \pm 3.1^{\mathrm{a}, \mathrm{b}}$ & $9.7 \pm 2.1^{\mathrm{a}}$ & $30.4 \pm 2.9^{\mathrm{a}}$ & $18.5 \pm 7.9^{\mathrm{a}}$ & $15.4 \pm 3.6$ & $15.6 \pm 3.1^{b}$ & $3.4 \pm 0.9^{\mathrm{a}, \mathrm{b}}$ \\
\hline $\mathrm{J}$ & -2.57 & 1.50 & $0.45 *$ & $22.9 \pm 5.6^{\mathrm{a}, \mathrm{b}}$ & $5.7 \pm 1.4^{b}$ & $19.9 \pm 2.6^{b}$ & $14.3 \pm 5.5^{b}$ & $20.1 \pm 4.9$ & $10.1 \pm 1.2^{\mathrm{a}}$ & $2.3 \pm 0.5^{a}$ \\
\hline $\mathrm{J}$ & -4.14 & 2.36 & $0.67 *$ & $20.1 \pm 2.0^{b}$ & $5.9 \pm 1.0^{b}$ & $22.6 \pm 2.0^{b}$ & $18.0 \pm 7.8^{\mathrm{a}}$ & $17.9 \pm 4.0$ & $12.2 \pm 2.4^{\mathrm{a}}$ & $2.5 \pm 0.7^{\mathrm{a}}$ \\
\hline Anual & -3.44 & 2.02 & $0.67 *$ & & & & & & & \\
\hline
\end{tabular}

Se indican las diferencias significativas con súper-índices (ANOVA). Relación alométrica ( $\left.\mathrm{Ms}=\mathrm{aLt} \mathrm{t}^{\mathrm{b}}\right)$; a; logaritmo de la constante de regresión: b; coeficiente de regresión: $r$; coeficiente de correlación: *; significativo $(\mathrm{P} \leq 0.05)$.

Significant differences in lower case super-indices (ANOVA). Allometric relationship ( $\mathrm{Ms}^{\mathrm{a}} \mathrm{aLt} \mathrm{L}^{\mathrm{b}}$; $\mathrm{a}$; logarithm of the regression constant: $\mathrm{b}$; regression coefficient: $r$; correlation coefficient: *; significant $(\mathrm{P} \leq 0.05)$. 
CUADRO 3

Valores promedio de los índices de condición por intervalos de longitud total de la concha y sexos de $A$. zebra en Chacopata, Venezuela

TABLE 3

Average values of condition indices intervals of total length of the shell and sexes of A. zebra in Chacopata, Venezuela

\begin{tabular}{|c|c|c|c|c|c|c|c|c|}
\hline $\begin{array}{l}\text { Intervalo } \\
\text { (mm) }\end{array}$ & $\mathrm{N}$ & $\mathrm{IC}_{1}$ & $\mathrm{IC}_{2}$ & $\mathrm{R}$ & IG & IM & $\mathrm{IC}_{3}$ & $\mathrm{IC}_{4}$ \\
\hline $40-49$ & 6 & $22.3 \pm 1.7$ & $10.2 \pm 1.1^{\mathrm{a}, \mathrm{b}}$ & $31.4 \pm 2.7^{\mathrm{a}}$ & $21.8 \pm 13.8$ & $11.5 \pm 2.8^{\mathrm{A}}$ & $9.5 \pm 3.6^{\mathrm{A}}$ & $2.2 \pm 0.9^{\mathrm{a}}$ \\
\hline $50-59$ & 25 & $20.3 \pm 2.9$ & $9.6 \pm 2.3^{\mathrm{a}}$ & $32.0 \pm 5.9^{\mathrm{a}}$ & $24.7 \pm 13.6$ & $13.2 \pm 3.9^{\mathrm{a}}$ & $12.7 \pm 5.1^{\mathrm{a}}$ & $2.5 \pm 0.8^{\mathrm{a}}$ \\
\hline $60-69$ & 86 & $21.5 \pm 3.9$ & $7.7 \pm 2.2^{b}$ & $26.1 \pm 5.0^{\mathrm{b}}$ & $17.7 \pm 10.2$ & $16.1 \pm 3.5^{\mathrm{a}}$ & $12.2 \pm 3.2^{\mathrm{a}}$ & $2.6 \pm 0.8^{\mathrm{a}}$ \\
\hline $70-79$ & 82 & $23.6 \pm 5.4$ & $7.6 \pm 2.5^{b}$ & $24.3 \pm 5.5^{\mathrm{b}}$ & $19.5 \pm 10.2$ & $16.4 \pm 4.4^{\mathrm{a}}$ & $13.2 \pm 3.6^{\mathrm{a}}$ & $3.1 \pm 0.9^{\text {a }}$ \\
\hline $80-89$ & 38 & $22.3 \pm 4.6$ & $8.3 \pm 3.1^{\mathrm{ab}}$ & $26.5 \pm 6.1^{b}$ & $17.1 \pm 10.6$ & $17.8 \pm 4.7^{b}$ & $16.1 \pm 4.1^{b}$ & $3.6 \pm 1.3^{\mathrm{b}}$ \\
\hline $90-99$ & & $22.4 \pm 2.4$ & $9.9 \pm 2.9^{\mathrm{a}, \mathrm{b}}$ & $30.4 \pm 6.2^{\mathrm{a}}$ & $13.3 \pm 9.7$ & $17.7 \pm 1.7^{\mathrm{B}}$ & $19.5 \pm 4.5^{\mathrm{B}}$ & $4.3 \pm 0.6^{\mathrm{b}}$ \\
\hline \multicolumn{9}{|c|}{ MACHOS } \\
\hline $40-49$ & 6 & $22.2 \pm 1.7$ & $10.2 \pm 1.1$ & $31.4 \pm 2.7$ & $21.8 \pm 13.8$ & $11.5 \pm 2.8$ & $9.5 \pm 3.6^{\mathrm{A}}$ & $2.2 \pm 1.0^{\mathrm{A}}$ \\
\hline $50-59$ & 18 & $20.5 \pm 2.3^{a}$ & $9.4 \pm 2.4$ & $31.0 \pm 4.8^{\mathrm{a}}$ & $22.6 \pm 11.7$ & $14.2 \pm 4.2^{\mathrm{a}}$ & $11.6 \pm 2.7^{\mathrm{a}}$ & $2.4 \pm 0.6^{\mathrm{a}}$ \\
\hline $60-69$ & 65 & $21.5 \pm 3.7^{\mathrm{a}}$ & $7.7 \pm 2.1$ & $26.2 \pm 4.6^{\mathrm{a}, \mathrm{b}}$ & $17.1 \pm 9.1$ & $16.2 \pm 3.5^{\mathrm{a}, \mathrm{b}}$ & $12.2 \pm 3.1^{\mathrm{a}}$ & $2.6 \pm 0.8^{\mathrm{a}}$ \\
\hline $70-79$ & 45 & $24.0 \pm 5.7^{b}$ & $7.7 \pm 2.3$ & $24.4 \pm 5.2^{\mathrm{a}, \mathrm{b}}$ & $18.9 \pm 10.4$ & $15.7 \pm 3.7^{\mathrm{a}, \mathrm{b}}$ & $13.4 \pm 3.4^{\mathrm{a}}$ & $3.1 \pm 0.9^{b}$ \\
\hline $80-89$ & 22 & $22.4 \pm 4.2^{\mathrm{a}, \mathrm{b}}$ & $9.0 \pm 3.5$ & $27.7 \pm 5.8^{\mathrm{a}, \mathrm{b}}$ & $18.2 \pm 11.0$ & $18.0 \pm 4.7^{\mathrm{b}}$ & $16.8 \pm 4.0^{b}$ & $3.8 \pm 1.4^{b}$ \\
\hline $90-99$ & 2 & $21.8 \pm 3.0$ & $11.3 \pm 2.4$ & $34.0 \pm 1.8$ & $17.7 \pm 8.4$ & $17.0 \pm 2.3^{\mathrm{B}}$ & $21.7 \pm 3.5^{\mathrm{B}}$ & $4.7 \pm 0.1^{\mathrm{B}}$ \\
\hline \multicolumn{9}{|c|}{ HEMBRAS } \\
\hline $50-59$ & 7 & $19.6 \pm 4.4$ & $10.2 \pm 2.2$ & $34.7 \pm 7.9$ & $30.2 \pm 17.4$ & $10.6 \pm 1.4^{*}$ & $15.4 \pm 8.3$ & $2.8 \pm 1.0$ \\
\hline $60-69$ & 21 & $21.8 \pm 4.9$ & $7.6 \pm 2.5$ & $25.7 \pm 6.2$ & $19.4 \pm 13.0$ & $15.7 \pm 3.5$ & $12.2 \pm 3.5$ & $2.6 \pm 0.9$ \\
\hline $70-79$ & 37 & $23.2 \pm 5.2$ & $7.5 \pm 2.7$ & $24.2 \pm 5.8$ & $20.4 \pm 10.0$ & $17.4 \pm 4.9$ & $13.1 \pm 3.8$ & $3.0 \pm 1.0$ \\
\hline $80-89$ & 16 & $22.2 \pm 5.2$ & $7.3 \pm 2.2$ & $24.8 \pm 6.3$ & $15.6 \pm 10.2$ & $17.6 \pm 4.7$ & $15.2 \pm 4.2$ & $3.3 \pm 0.9$ \\
\hline
\end{tabular}

Se indican las diferencias significativas con superíndices en minúsculas (ANOVA) y mayúscula (KS).

Superscript lower case (ANOVA) and upper case (KS) significant differences are indicated.

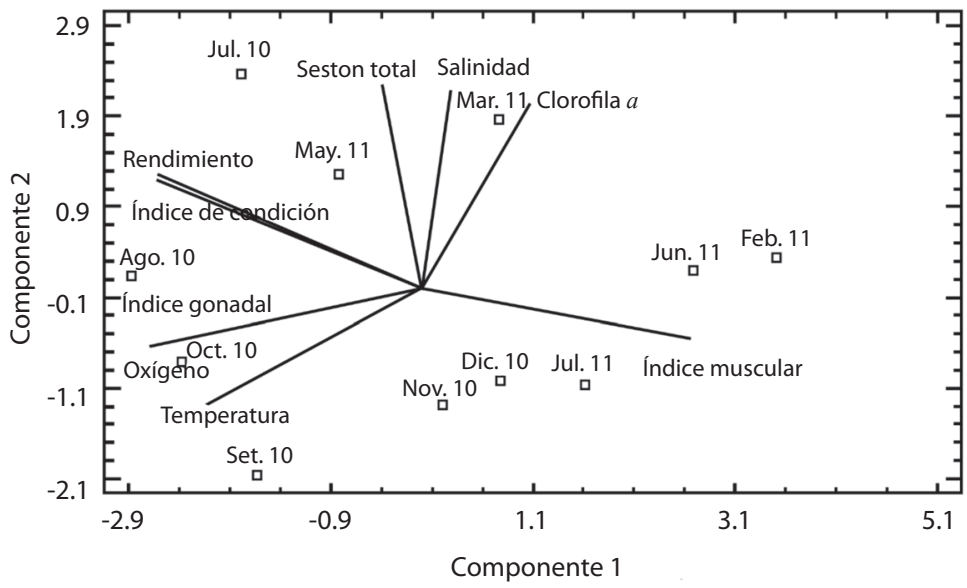

Fig. 1. Proyecciones ortogonales del Análisis de Componentes Principales (ACP) de las variables ambientales y los índices de condición de Arca zebra.

Fig. 1. Principal Component Analysis (PCA) of environmental variables and condition indices of Arca zebra. 
sin embargo, no se manejan criterios sólidos de estimación de su valor económico por la calidad de la carne, que está constituida en una buena proporción por la gónada que se extiende desde la glándula digestiva entremezclada con los tejidos musculares y órganos que la hacen atractiva para su explotación y consumo, cuando alcanzan el máximo desarrollo gonádico.

La clorofila $a$, el seston total y la salinidad, no mostraron una asociación positiva con los índices fisiológicos, sin embargo, en marzo, mayo y julio 2011, se asociaron con la productividad primaria de los periodos de surgencia, y del incremento de la productividad en julio, que ocurre localmente y es asociada a desoves secundarios en algunos bivalvos. Mientras que en el ACP, la baja temperatura fue el factor con mejor relación con los índices de masas (IC, IG, R), como se puede apreciar en los promedios mensuales más bajos durante los primeros meses del año (que se asocian a la surgencia costera), mientras que entre las tallas (40-59 mm Lt), estos índices tienen los promedios más altos, lo que sugiere que a partir de $60 \mathrm{~mm}$ Lt sus valores disminuyen asociados a la liberación de gametos. Por el contrario, el índice muscular (IM) y los índices basados en la longitud de la concha $\left(\mathrm{IC}_{3}, \mathrm{IC}_{4}\right)$, no presentaron diferencias significativas entre los meses (por tratarse de un grupo de ejemplares que pertenecen a un grupo etario uniforme). Sin embargo, los incrementos entre las tallas $(\mathrm{P} \leq$ 0.05 ) indican que son proporcionales al tamaño del individuo, e independientes de la condición fisiológica y de productividad primaria. De tal forma que, los índices de condición fisiológica basados en los tejidos blandos van a reflejar principalmente diferencias estacionales del estadio reproductivo y de acumulación de reservas energéticas, sin diferencias significativas entre sexos, y en relación inversa a las tallas debido al mayor gasto energético por la liberación de gametos.

La ecuación de alometría es uno de los modelos por excelencia para estimar la condición fisiológica de una población, sin embargo, en este trabajo no se obtuvieron relaciones significativas por el estrecho rango de los datos examinados. Sin embargo, en esta especie el incremento en los tejidos blandos y concha es proporcional, como ha sido señalado por Prieto et al. (2001) y Aponte et al. (2008). Mientras que los índices de condición alcanzan los promedios más altos con buena disponibilidad del seston, Prieto y Acosta (2008) sugieren que los bivalvos pueden aprovechar el detritus orgánico para suplir su metabolismo básico, como fuente alternativa de alimento.

El índice muscular (IM) de Pectínidos y Pteroideos tiende a ser alto y representa la principal medida de la condición fisiológica de las poblaciones. Estos organismos poseen un músculo prominente, de textura blanda y alto contenido de glucógeno, que es acumulado y transferido a las gónadas para el ciclo gametogénico que tiende a estar en relación inversa al IG, como ha sido reportado en Atrina maura, Nodipecten nodosus, Argopecten ventricosus, Nodipecten subnodosus y Spondylus princeps (Villalejo \& Ceballos, 1996; Villalejo, Tripp, \& García, 2005; Pérez, Serrano, \& Ahumada, 2007; García et al., 2007). Por el contrario, el músculo de $A$. zebra es rígido, su tamaño y masa incrementan proporcionalmente a la concha, que descartan tenga un rol en la bioenergética reproductiva y de producción de carne. La dinámica poblacional de $A$. zebra estuvo asociada principalmente al contenido de carne y gónadas durante el período de estudio, por lo que es recomendable evaluar individualmente la glándula digestiva y su relación con los procesos de crecimiento y maduración gonadal.

Los índices de condición en ambos sexos, presentaron comportamientos similares, que sugieren la sincronía de la actividad reproductiva en la especie, y muestra una estrategia reproductiva oportunista, que aprovecha la disponibilidad de nutrientes para realizar los desoves. Esto garantiza la supervivencia de la especie, ya que la liberación de los gametos en los períodos de alta productividad primaria, facilitaría el asentamiento de las larvas y el establecimiento continuo de individuos, que remplazarían a los que son extraídos por pesca, mortalidad natural y depredación. 
En conclusión, la dinámica poblacional de A. zebra se puede explicar por las variaciones de los índices de condición basados en las masas de los tejidos blandos. En esta especie los incrementos de la masa de los tejidos blandos son proporcionales a las dimensiones de la concha, de modo que la extracción del recurso presentará los mayores rendimientos en carne los meses de mayor temperatura del agua y/o mientras la especie no desove. La concha representó una variable robusta para estimar el provecho del organismo; mientras se mantengan los cánones de extracción, se puede asegurar la conservación de la especie y una pesquería sostenible en el tiempo, así mismo la obtención de reproductores para criaderos de semillas con fines de cultivo e investigación.

\section{AGRADECIMIENTOS}

Al Consejo de Investigación de la Universidad de Oriente, por el financiamiento del proyecto: "Variación estacional de la composición bioquímica de la pepitona Arca zebra, en relación al estadio reproductivo y los factores ambientales", subvencionado por el proyecto ( ${ }^{\circ}$ CI-0203060 I-1777-12).

\section{RESUMEN}

Los índices de condición en sus diferentes versiones reflejan el desempeño del organismo en su ambiente, así como la condición somática, el valor nutritivo, la importancia comercial, el papel ecológico, y periodos de engorde. En este estudio se evaluaron diferentes índices fisiológicos, con la finalidad de conocer el estado de la población de Arca zebra y proponer estrategias de explotación y conservación con fines de consumo del recurso. Se recolectaron un total de 240 ejemplares entre julio 2010-julio 2011, en el banco de Chacopata. Mensualmente se midió la longitud total, altura y grosor de la concha. Los tejidos blandos fueron deshidratados para obtener la masa seca total. Se analizaron diferentes índices de condición: los basados en la masa del organismo, los que combinan masa y longitud de la concha, índice gonadal, índice muscular, rendimiento de la carne, la masa fresca de los tejidos blandos en relación a la longitud total y la masa seca de los tejidos blandos en relación a la longitud total. El modelo de crecimiento fue alométrico positivo entre la longitud-masa seca total. El peso de la gónada ejerció una marcada influencia sobre el índice de condición, índice gonadosmático y rendimiento.
La población estuvo conformada por 82 machos y 158 hembras. Los incrementos de la masa de los tejidos blandos fueron proporcionales a las dimensiones de la concha. Se propone el índice que contrasta la masa fresca de los tejidos blandos en relación a la longitud total, como una herramienta que le permitiría a los pescadores a estimar la dinámica de la población de $A$. zebra, la cual ayudaría a ejercer una pesquería sustentable en el tiempo, mientras se mantengan los cánones de extracción.

Palabras clave: Índice de condición, dinámica poblacional, desoves, acumulación de reservas.

\section{REFERENCIAS}

Acosta, V., Prieto, A., \& Lodeiros, C. (2006). Índice de condición de los mejillones Perna perna y Perna viridis (Bivalvia: Mytilidae) bajo un sistema suspendido de cultivo en la Ensenada de Turpialito, Golfo de Cariaco, Venezuela. Zootecnia Tropical, 24(2), 177-192.

Acosta, V., Prieto, A., Licett, B., Longart, Y., \& Montes, M. (2011). Rendimiento, índice de condición e índice gonadal del mejillón verde Perna viridis en cultivo de fondo en el Golfo de Cariaco, estado Sucre, Venezuela. Zootecnia Tropical, 29(4), 399-410.

Aponte, A., Prieto, A., \& Lemus, M. (2008). Relación longitud-peso seco de la pepitona Arca zebra (Swainson, 1833) procedente de la costa norte de la Península de Araya, Estado Sucre, Venezuela. Boletín del Instituto Oceanográfico de Venezuela, Universidad de Oriente, (1), 59-65.

Barber, B., \& Blake, N. (1991). Reproductive physiology. En S. Shumway (Ed.), Scallops: Biology, Ecology and Aquaculture, Developments in Aquaculture and Fisheries Science (Vol. 21, pp. 377-428) Elselvier Science Publishers B. V.

Cruz, R. (1982). Variación mensual del índice de condición del molusco Anadara tuberculosa (Pelecypoda: Arcidae) en Punta Morales, Puntarenas, Costa Rica. Revista de Biología Tropical, 30(1), 1-4.

Davenport, J., \& Chen, X. (1987). A comparison of methods for the assessment of condition in the mussel (Mytilus edulis L.). Journal of Molluscan Studies, 53, 293-297.

García, N., Lodeiros, C., Arrieche, D., Prieto, A., Freites, L., \& Himmelman, J. (2007). Relación del tejido somático y los factores ambientales en el ciclo reproductivo de la vieira (Nodipecten nodosus). Boletín del Centro de Investigaciones Biológicas, 41(3), 292-308.

Harding, J. P. (1949). The use of probability paper for the graphical analysis of polymodal frequency 
distributions. Journal of the Marine Biological Association UK, 28, 141-153.

Hickman, R. E., \& Illingworth, J. (1980). Condition cycle of the green lipped mussel Perna canaliculus in New Zealand. Marine Biology, 60, 27-38.

Jiménez, R. (1999). Análisis y evaluación del recurso pepitona, Arca zebra en el banco de Chacopata. Memorias del taller Venezolano sobre aprovechamiento y comercialización de moluscos bivalvos, Isla de Margarita, Venezuela.

Kagley, A. N., Snider, R. G., Krishnakumar, P. K., \& Casillas, E. (2003). Assessment of Seasonal Variability of Cytochemical Responses to Contaminant Exposure in the Blue Mussel Mytilus edulis (Complex). Archives of Environmental Contamination and Toxicology, 44, 43-52.

Lista, M., Lodeiros, C., Prieto, A., Himmelman, J., Castañeda, J., García, N., \& Velásquez, C. (2006). Relation of seasonal changes in the mass of the gonad and somatic tissues. Journal of Shellfish Research, 25, 969-973.

Lista, M., Prieto, A., Velásquez, C., Lodeiros, C., Acosta, V., Longart, Y., \& Hernández, G. (2011). Descripción y variación mensual de las etapas reproductivas de la pepitona Arca zebra (Swainson, 1833) en el banco de Chacopata, península de Araya, estado Sucre, Venezuela. Zootecnia Tropical, 29(1), 89-102.

Lucas, A., \& Beninger, P. G. (1985). The use of physiological condition indices in marine bivalve aquaculture. Aquaculture, 44, 187-200.

Martin, M., Ichikawa, G., Goetzl, J., Reyes, M., \& Stephenson, M. D. (1984). Relationships Between Physiological Stress and Trace Toxic Substances in the Bay Mussel, Mytilus edulis, from San Francisco Bay, California. Marine Environment Research, 11, 91-110.

Mendoza, J. (2008). Situación actual y perspectivas de las pesquerías marítimas en Venezuela. Recursos Marinos Acuícolas, 2, 121-137.

Ministerio de Agricultura y Tierras. (1960). Instituto Nacional de la Pesca y Acuicultura. Resolución MAC-RNR-266. Gaceta Oficial de la República Bolivariana de Venezuela $N^{\circ} 195,361$.

Nascimento, I., \& Pereira, S. (1980). Changes in the condition index from mangrove oyster (Crassostrea rhizophorae) from Todos Los Santos Bay, Salvador, Brazil. Aquaculture, 20, 9-15.

Pérez, C., Serrano, S., \& Ahumada, M. (2007). Ciclo reproductivo del molusco Atrina maura (Pterioidea: Pinnidae) en un sistema lagunar costero, al sur del Pacífico tropical mexicano. Revista de Biología Tropical, 55, 839-852.

Prieto, A., Ramos, O., Arrieche, D., Villalba, J., \& Lodeiros, C. (2001). Producción secundaria e índice de condición en Arca zebra (Mollusca: Bivalvia) del Golfo de Cariaco, Venezuela. Revista de Biología Tropical, 49(2), 599-608.

Prieto, A., \& Acosta, V. (2008). Producción secundaria de una población cultivada del mejillón verde (Perna viridis) en el golfo de Cariaco, Venezuela. Boletín del Centro de Investigaciones Biológicas, 21, 38-45.

Salaya, J. (1999). La pesca y cultivo de moluscos bivalvos en Venezuela, situación y tendencias a nivel de Latinoamérica y el Caribe (pp. 5-11). Memorias Taller "Aprovechamiento y comercialización de moluscos bivalvos". Facultad de Ciencias, Universidad Central de Venezuela, Margarita, Venezuela.

Sarkis, S. (1992). The turkey-wing mussel, Arca zebra: Aspects of its Ecology, Reproduction and Physiology in Bermudan Waters (PhD Thesis). Polytechnic South West, Bermuda.

Sarkis, S. (1993). Seasonal changes in the gross biochemical composition of the turkey-wing Arca zebra, in Bermuda. Journal of Shellfish Research, 12(2), 329-336.

Sokal, R., \& Rohlf, F. (1969). Biometry. The principles and practices of statistics in biological research. San Francisco: W.H. Freeman.

Strickland, J., \& Parsons, T. (1972). A practical handbook of seawater analysis. Bulletin Fisheries Research, $16,167-315$.

Villalejo, M., \& Ceballos, P. (1996). Variación de los índices de condición general, gonádico y de rendimiento muscular en Argopecten circularis (Bivalvia: Pectinidae). Revista de Biología Tropical, 44, 591-594.

Villalejo, M., Tripp, A., \& García, F. (2005). Variación de los índices gonádico, de rendimiento muscular y de la glándula digestiva de Spondylus princeps (Gray, 1825) (Mollusca: Bivalvia) en Isla Cedros y Punta Eugenia, México. Revista Biología Marina y Oceanografia, 40, 87-90. 